\title{
Accurate and efficient data processing for quantitative real-time PCR using a tripartite plant virus as a model
}

\author{
Junli Feng ${ }^{1,2}$, Rong Zeng2, and Jishuang Chen ${ }^{1,2}$
}

BioTechniques 44:901-912 (June 2008)

doi $10.2144 / 000112750$

\begin{abstract}
Real-time PCR is becoming a preferred method for quantification of minute amounts of nucleic acids. To achieve the full potential of this technique, accurate and convenient models for post-PCR data analysis are required. In this study, three different models were chosen to quantify the definitive copy numbers of Cucumber mosaic virus (CMV) genomic RNAs using raw fluorescence data of real-time PCR, and equations were proposed to compare their expression levels in virions or in planta. The results, as confirmed by standard curve and Northern blotting methods, show that the expression levels of different genes can be compared more accurately and more efficiently by these equations, especially using theoretical fluorescence $\left(\mathrm{F}_{0}\right)$ and calibration factors $(\mathrm{CF})$, determined by linear regression PCR (LinRegPCR). Thus, these equations, combined with data analysis by the LinRegPCR method, can greatly enhance the high-throughput quantification ability of real-time PCR, and permit accurate, reliable, and facile investigation of the changes in CMV RNAs accumulation.
\end{abstract}

\section{INTRODUCTION}

Recent developments have made real-time PCR a powerful technology for detection and quantification of nucleic acids $(1,2)$. With a high degree of sensitivity and a large dynamic range, it has greatly benefited many aspects of biological research, including plant virology. However, the full potential of this technology has yet to be realized, partially because of the limitations of the calculation methods that currently predominate (3).

The large quantities of numerical data produced by real-time PCR are generally analyzed by basic software tools provided with the PCR fluorescent thermal cyclers, which quantify the unknown samples either relatively or absolutely by comparing them to the standard DNA or to the reference gene. These two groups of methods are based on the same assumptions, namely, that amplification efficiencies of target genes are approximately equal to the standard DNA or the reference genes, and that the amplification efficiency is constant throughout the PCR (4). However, as noted by many researchers, this is often not the case in practical applications and it is believed to be a non-negligible source of biased results $(5,6)$.

To circumvent the amplification efficiency problem, several new mathematical models have recently emerged for analyzing PCR data. By investigating the raw PCR fluorescence value measured per cycle of each reaction, these models directly predict the theoretical fluorescence value at cycle $0\left(F_{0}\right)$. As the basic principle underlying real-time PCR is the fluorescence intensity proportional to DNA content, once $F_{0}$ is determined, the changes in the amount of target genes can be represented by the changes of $F_{0}$ values. However, using only $F_{0}$ values is inadequate when the definitive copy numbers are required or when expression levels of different genes are compared. The reason for this is that the calibration factor $(C F)$ of the target gene, which relates fluorescence to DNA molecular content in realtime RT-PCR, is unknown. Nevertheless, these problems can be solved through amplification of template with known copy numbers (7).

Only one study to date has reported the determination of $C F$ values, which used sigmoidal curve-fitting (SCF), one of the most popular fluorescence-based mathematical models (3). Therefore, in the present study, the determination of $C F$ values was applied to two other more facile models: linear regression PCR (LinRegPCR) and data analysis for realtime PCR (DART). This was done by quantifying and comparing the expression levels of three genes $(1 a, 2 a$, and $M P)$ of Cucumber mosaic virus (CMV), an important plant virus that is known to infect over 1000 plant species and is spread worldwide (8). The $l a, 2 a$, and $M P$ genes were chosen because their amounts could be used to represent the accumulation levels of CMV genomic RNAs 1, 2, and 3 , respectively. For different strains of CMV and at different stages of infection, the accumulation levels of RNAs and their ratios were changed, and these changes were regarded as the result of interaction between virus, host plant, and environment. 


\section{Research Reports}

In our previous work, the expression levels of CMV RNAs of different strains in host plants were quantified relatively using a reference gene (18S rRNA), but the relative comparison between them could not be accomplished due to the different PCR efficiencies $(9,10)$. Accordingly, in this study, we proposed equations to compare the expression levels of CMV RNAs 1, 2, and 3 by investigating the fluorescence kinetics of each individual reaction. To test their reliability and to allow a comparison, the expression ratios were also quantified by the standard curve method and by traditional Northern blotting.

\section{MATERIALS AND METHODS}

\section{Plants, Viruses, and Standard Samples}

Seedlings of tobacco (Nicotiana tobacum $c v$. Huangmiaoyu) were used as host plants and grown in a greenhouse between $21-26^{\circ} \mathrm{C}$. The wild-type Fny$\mathrm{CMV}$ and four intraspecies hybrid viruses FCb72b-CMV, FRad352b-CMV, FBX 2b$\mathrm{CMV}$, and $\mathrm{FNa}^{2 \mathrm{~b}} \mathrm{CMV}$ were generated as described (11). All viral strains were inoculated with the same concentrations on tobacco seedlings at the four- to sixleaf stage. At 30 days post-inoculation (dpi), virions were purified from $5 \mathrm{~g}$ fresh leaf tissues.

Another set of tobacco plants was inoculated for detection of the accumulation of CMV genomic RNAs in plant tissues with presence or absence of T1satellite (T1sat). At 4 dpi, $0.2 \mathrm{~g}$ fresh leaf tissues were sampled from systemic leaves of infected plants. Two biological repeats were conducted for each treatment.

Standard samples of $l a, 2 a$, and $M P$ genes were transcribed in vitro from biologically active full-length cDNA clones of Fny-CMV RNAs as described in a previous study (9). Before use, the concentration of each transcription product was determined by a spectrophotometer (SpectraMax Plus 384; Molecular Devices, Sunnyvale, CA, USA) and serial dilutions were performed to give final concentrations of between $2 \times 10^{9}$ to $2 \times$ $10^{5}$ copies $/ \mathrm{mL}$.

\section{RNA Extraction and Reverse Transcription}

RNAs were extracted from purified virions or plant tissues using TRIzol reagent (Invitrogen, Carlsbad, CA, USA), and treated with RNase-free DNase to remove any traces of genomic DNA contamination. The extract integrity was electrophoretically verified. Viral RNAs and standard RNAs $(1 \mu \mathrm{l})$ were reverse-transcribed with $100 \mathrm{U}$ of AMV (Takara, Dalian, China) using $100 \mu \mathrm{M}$ random hexamer primers according to the manufacturer's instructions.

\section{Real-time PCR Assay}

The SYBR Green PCR was performed on 96-well optical plates using an ABI Prism 7300 Sequence Detection System (PE Applied Biosystems, Foster City, CA, USA). Primers used to detect $l a, 2 a$, and $M P$ genes were optimized as per our previous publication (9). For each $25 \mu \mathrm{l}$ reaction volume, $12.5 \mu$ l Premix $E X$ Taq ( $2 \times$; Takara), $0.5 \mu \mathrm{l}$ SYBR Green I nucleic acid fluorescent dye (50× ; Takara), $2 \mu \mathrm{l}$ template cDNA, $1 \mu \mathrm{l}$ forward and reverse primers $(1 \mu \mathrm{M})$, and $9 \mu \mathrm{l}$ PCR-grade water were mixed. The parameters for a two-step PCR were initiated by incubation at $95^{\circ} \mathrm{C}$ for $10 \mathrm{~s}$, followed by 40 cycles of $95^{\circ} \mathrm{C}$ for $10 \mathrm{~s}$ and $60^{\circ} \mathrm{C}$ for $30 \mathrm{~s}$. Each reaction was repeated three times and the RNA extracted from healthy tobacco plants was used as a negative control. The specificity of the amplified products was examined by melting curve analysis immediately after the final PCR cycle.

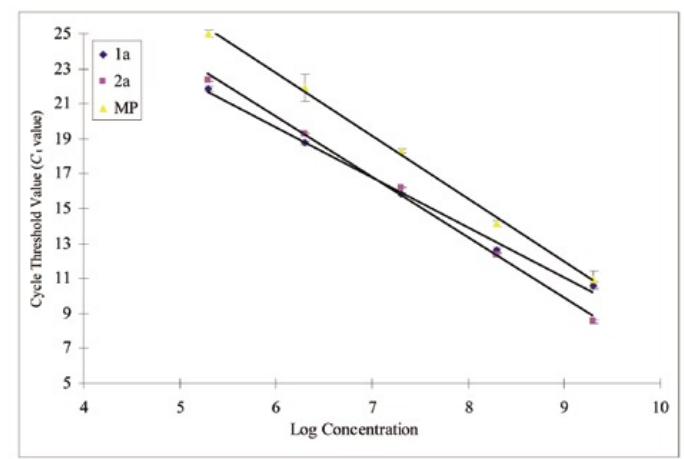

Figure 1. Standard curves of Cucumber mosaic virus (CMV) genes. The average $C_{T}$ values were plotted vs. $\log$ concentration of standard samples of CMV $1 a, 2 a$, and MP genes. The error bars indicate the standard deviations obtained from three independent experiments. $1 a: \mathrm{Y}=-2.866 \mathrm{X}+36.862\left(\mathrm{R}^{2}=\right.$ 0.995); $2 a: \mathrm{Y}=-3.459 \mathrm{X}+41.008\left(\mathrm{R}^{2}\right.$ $=0.997) ; M P: \mathrm{Y}=-3.595 \mathrm{X}+44.312$ $\left(\mathrm{R}^{2}=0.998\right)$.

Table 1. Correlation of Reaction Fluorescence Values to Mass of Standard RNAs Representing RNAs 1, 2, and 3 of Cucumber mosaic virus (CMV)

\begin{tabular}{|c|c|c|c|c|c|c|c|c|c|}
\hline \multicolumn{4}{|c|}{$1 \mathrm{a}(72 \mathrm{bp})$} & \multicolumn{3}{|l|}{$2 a(76$ bp) } & \multicolumn{3}{|l|}{ MP (154 bp) } \\
\hline Predicted $\mathrm{N}_{0}^{\mathrm{a}}$ & $C F_{\text {SCF }^{b}}$ & $C F_{\text {LinReg }}{ }^{c}$ & $C F_{\mathrm{DART}}{ }^{\mathrm{d}}$ & $C F_{\mathrm{SCF}^{\mathrm{b}}}$ & $C F_{\text {LinReg }}{ }^{c}$ & $C F_{\mathrm{DART}}{ }^{\mathrm{d}}$ & $C F_{\text {SCF }^{b}}$ & $C F_{\text {LinReg }}{ }^{\mathrm{c}}$ & $C F_{\mathrm{DART}}{ }^{\mathrm{d}}$ \\
\hline $2 \mathrm{E}+09$ & 64.6 & 44.2 & 40.8 & 65.0 & 40.0 & 39.5 & 75.2 & 43.6 & 46.0 \\
\hline $2 \mathrm{E}+08$ & 54.4 & 39.7 & 32.4 & 70.8 & 41.7 & 43.7 & 65.6 & 47.7 & 48.3 \\
\hline $2 \mathrm{E}+07$ & 67.6 & 42.0 & 38.0 & 66.3 & 46.7 & 44.5 & 65.0 & 54.0 & 41.3 \\
\hline $2 \mathrm{E}+06$ & 61.8 & 50.4 & 48.4 & 65.5 & 46.5 & 35.8 & 64.2 & 44.7 & 52.8 \\
\hline $2 \mathrm{E}+05$ & 57.8 & 43.2 & 47.1 & 61.6 & 45.6 & 48.8 & 71.7 & 49.4 & 56.9 \\
\hline Average $/ \pm \mathrm{CV}$ e & $61.2 \pm 8.62 \%$ & $43.9 \pm 9.14 \%$ & $41.3 \pm 15.93 \%$ & $65.8 \pm 4.98 \%$ & $44.1 \pm 6.98 \%$ & $42.5 \pm 11.79 \%$ & $68.3 \pm 7.11 \%$ & $47.9 \pm 8.63 \%$ & $49.1 \pm 12.28 \%$ \\
\hline $\begin{array}{l}\text { aPredicted input an } \\
\text { bSigmoidal curve-fi } \\
\text { 'Linear regression } \\
\text { dData analysis for } \\
\text { eAverage } \pm \text { coeffici }\end{array}$ & $\begin{array}{l}\text { ount of target mol } \\
\text { ting (SCF)-based } \\
\text { PCR (LinRegPCR) } \\
\text { eal-time PCR (DA) } \\
\text { ent of variation. }\end{array}$ & $\begin{array}{l}\text { cules based upon } \\
\text { alibration factor ( } \mathrm{n} \\
\text { based calibration } \\
\text { T)-based calibrati }\end{array}$ & $\begin{array}{l}\text { dilution of standard } \\
\text { /FU). } \\
\text { actor (ng/FU). } \\
\text { n factor (ng/FU). }\end{array}$ & & ed via $A_{260}$. & & & & \\
\hline
\end{tabular}




\section{Research Reports}

\section{Absolute Quantification by Standard Curve Method}

The PCR data were initially analyzed using Sequence Detection Software (PE Applied Biosystems), and resulted in the assignation of a threshold cycle $\left(\mathrm{C}_{\mathrm{T}}\right)$ value to each reaction. For each target gene, a standard curve was created based on the linear relationship between the $\mathrm{C}_{\mathrm{T}}$ values of standard samples and the logarithm of the starting copy numbers, which were derived from the concentration of standard samples by Equation 1:

$$
\mathrm{N}=\mathrm{C} /\left(\mathrm{K} \times 330 \times 1.6601 \times 10^{-18}\right),
$$

[Eq. 1]

where $\mathrm{N}$ is the copy number per $\mu \mathrm{L}, \mathrm{C}$ is the concentration per sample $(\mu \mathrm{g} / \mu \mathrm{L}), \mathrm{K}$ is the length of each target gene (bases), and $1.6601 \times 10^{-18}$ is the conversion constant between Dalton and $\mu \mathrm{g}$.

For the unknown samples, copy numbers of each gene were provided by the software according to the mean of the standard curve and their respective $\mathrm{C}_{\mathrm{T}}$ values.

\section{Real-time PCR Fluorescence Data Analysis by SCF}

After real-time PCR, the fluorescence data of each product were exported to an Excel workbook for analysis. Using Origin 7.5, the fluorescence readings of individual PCR amplifications were fitted to the nonlinear regression function given by Equation $2(3,12)$ :

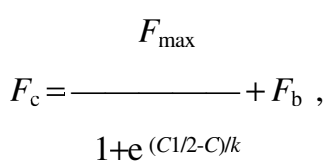

[Eq. 2]

where $C$ is the cycle number, $F_{\mathrm{c}}$ is the reaction fluorescence at cycle $C, F_{\max }$ is the maximal fluorescence during the reaction, $\mathrm{e}$ is the natural logarithm base, $C_{1 / 2}$ is the cycle at which reaction fluorescence reaches half of $F_{\text {max }}, k$ is the slope of the sigmoid curve, and $F_{\mathrm{b}}$ is the background reaction fluorescence.

In contrast to $\mathrm{C}_{\mathrm{P}}$ values in the standard curve method, the initial copy numbers of target genes $\left(N_{0}\right)$ were represented directly by $F_{0}$ values in the SCF method, which are given by a simple derivation of Equation 2, when $C=0$ :

$$
F_{0}=\frac{F_{\text {max }}}{1+\mathrm{e}^{C 1 / 2 / k}} .
$$

[Eq. 3]

Figure 2. Quantification of the copy number of Cucumber mosaic virus (CMV) genes in virions. Copy numbers of each gene in five types of virions were calculated by (A) standard curves, (B) sigmoidal curve-fitting, (C) LinRegPCR, and (D) DART methods, based on the $\mathrm{C}_{\mathrm{T}}$ values for standard curve method or $F_{0}$ values of all the other calculation methods. Real-time PCR experiments were repeated three times, and variations between the results of the three experiments were reported as the standard deviation ( \pm SD).

B

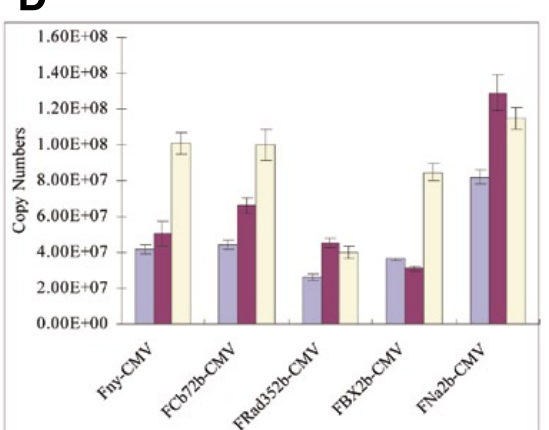

\begin{tabular}{|c|c|c|c|c|c|}
\hline \multirow{2}{*}{$\begin{array}{l}\text { Calculation } \\
\text { Methods }\end{array}$} & \multicolumn{5}{|c|}{ Copy Number Ratiosa } \\
\hline & Fny-CMV & FCb72b-CMV & FRad352b-CMV & FBX2b-CMV & FNa ${ }^{2 b}-\mathrm{CMV}$ \\
\hline Standard curve & 1:1.34:2.36 & $1: 1.37: 2.17$ & 1:1.39:1.47 & $1: 0.80: 2.20$ & 1:1.38:1.65 \\
\hline LinRegPCR & 1:1.35:2.39 & 1:1.46:2.69 & $1: 1.60: 1.51$ & $1: 0.75: 2.26$ & $1: 1.43: 1.62$ \\
\hline DART & $1: 1.20: 2.43$ & $1: 1.50: 2.26$ & $1: 1.71: 1.53$ & $1: 0.86: 2.34$ & $1: 1.57: 1.40$ \\
\hline
\end{tabular}

Table 2. Comparison of the Accumulation Ratios of Cucumber mosaic virus (CMV) RNAs 1, 2, and 3 in Virions of Five CMV Strains Determined by Various Methods 


\section{Research Reports}
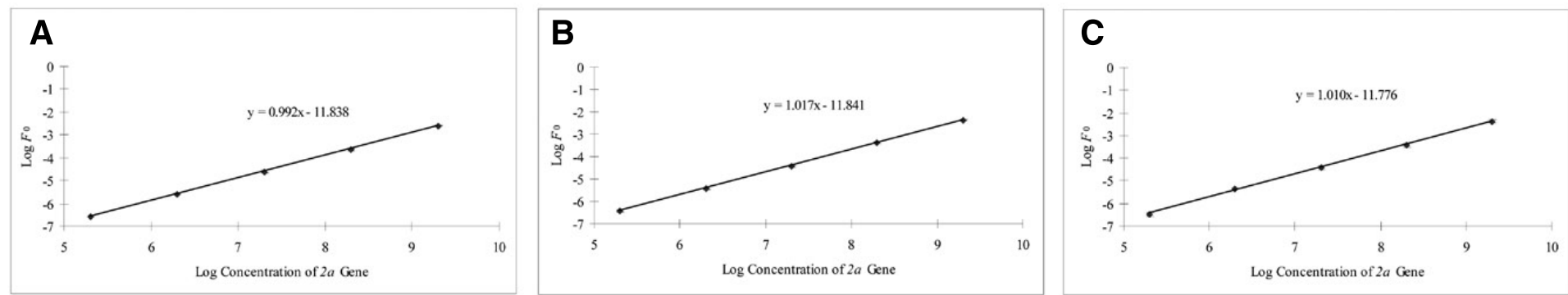

Figure 3. The concentration of the $2 a$ gene presented by $\boldsymbol{F}_{\mathbf{0}}$ values. The linear regression analysis generated by plotting the log of copy numbers of the $2 a$ gene vs. $\log$ of $F_{0}$, which was determined by (A) sigmoidal curve-fitting, (B) LinRegPCR, and (C) DART methods. The nearly perfect linear relationship (R ${ }^{2} \geq$ 0.999 ) indicates that $F_{0}$ directly reflects the concentration of $2 a$ gene. The results correspond to triplicate experiments.

When the fluorescence dataset of each product was fitted to Equation 2, a series of $r^{2}, F_{\max }, C_{1 / 2}$, and $k$ values was generated and $F_{0}$ values were obtained. The minimum-calculated $F_{0}$ value, which was produced based upon the criterion recommended by Rutledge (3), was used to reflect the initial target quantity. Once the $F_{0}$ value is determined, it can be converted to $N_{0}$ by Equation 4 :

$$
N_{0}=\left(C F_{\mathrm{SCF}} \times F_{0} \times 9.1 \times 10^{11}\right) / \mathrm{A}_{\mathrm{s}},
$$

where $C F_{\mathrm{SCH}}$ is the SCF-based calibration factor, expressed as the number of nanograms of double-stranded DNA per SYBR Green I fluorescence unit (ng/FU), $\mathrm{A}_{\mathrm{s}}$ is the product size in base pairs, and $9.1 \times 10^{11}$ is the number of single base pair molecules per nanogram. It should be noted that $C F_{\mathrm{SCF}}$ determines the absolute accuracy or "exactness" of quantitative scale in SCF methods (3), and it can be determined straightforwardly by the $F_{0}$ value of standard samples of known concentration, based upon rearrangement of Equation 4:

$$
C F_{\mathrm{SCH}}=\left(N_{\mathrm{P}} \times \mathrm{A}_{\mathrm{s}}\right) /\left(F_{0} \times 9.1 \times 10^{11}\right)
$$

where $N_{\mathrm{P}}$ is the predicted copy number of target genes in standard samples. As long as the $C F_{\mathrm{SCH}}$ value of a specific gene is determined, the calculation of $N_{0}$ values of this gene in an unknown sample simply requires the knowledge of the $F_{0}$ value, which can be derived from the raw fluorescence databased on Equation 3.

\section{Real-time PCR Fluorescence Data Analysis by LinReg and DART}

The exported PCR data were also analyzed by the LinRegPCR 7.5 program (13) and by the DART-PCR version 1.0 program (14). The LinRegPCR program is based on the basic exponential formula of PCR amplification (15):

$$
X_{\mathrm{c}}=X_{0} \times(E)^{c},
$$

[Eq. 6]

where $X_{\mathrm{c}}$ is the concentration of template at cycle $c, X_{0}$ is the initial concentration of template, and $E$ is the amplification efficiency ranging from 1 to 2 . This equation can be expressed linearly in terms of fluorescence by taking the logarithm of both sides:

$$
\log \left(F_{\mathrm{c}}\right)=\log \left(F_{0}\right)+\log (E) \times C,
$$

[Eq. 7]

where $F_{\mathrm{c}}$ and $C$ are measured fluorescence data and cycle number, respectively. For each amplification curve, the log-linear part of the PCR was automatically determined by the program with the default option to contain from four to six points with the highest $R^{2}$ value. Once these points were determined, they were fitted to a regression line and the initial fluorescence $F_{0}$ then directly calculated as $10^{\text {intercept. }}$

The DART program is also based on Equation 6, but with another reformulation:

$$
F_{0}=F_{\mathrm{CT}} \times(E)^{-\mathrm{CT}},
$$

where $F_{\mathrm{CT}}$ is the fluorescence at threshold cycle. As described by Peirson et al. (14), using the raw fluorescence data, this program can calculate the individual $\mathrm{C}_{\mathrm{l}}$, average $E$ values, and the resulting $F_{0}$ values automatically and rapidly.

For both LinReg and DART methods, once the $F_{0}$ value of each gene in a series of diluted standard samples is obtained, their respective $C F_{\text {LinReg }}$ and $C F_{\text {DART }}$ value can be determined by Equation 5 . The $N_{0}$ values of CMV $1 a, 2 a$, and $M P$ genes in unknown samples can then be calculated in the same way as for the SCF method.

\section{Relative comparison of CMV RNAs $\operatorname{using} N_{0}$}

Once the $N_{0}$ values of CMV genes are obtained, their relative expression levels in the same RNA samples can be compared directly:

$$
\text { Ratio }_{\mathrm{A} / B}=N_{\mathrm{OA}} / N_{\mathrm{OB}}=\frac{C F_{\mathrm{A}} \times F_{\mathrm{OA}} \times \mathrm{A}_{\mathrm{SB}}}{C F_{\mathrm{B}} \times F_{\mathrm{OB}} \times \mathrm{A}_{\mathrm{SA}}} .
$$

[Eq. 9]

However, when their copy numbers in different samples are compared, the variances in amounts of total RNA should be taken into account, which can be represented by changes of the $F_{0}$ values of the reference gene (18S rRNA) between samples. Therefore, the above formula was rearranged to:

$$
\text { Ratio }_{\text {Asample1/Bsample2 }}=\frac{N_{0 \text { A sample1 }} / N_{0 \text { B sample2 }}}{F_{0 \text { Refsample1 }} / F_{0 \text { Refsample2 }}} .
$$




\section{Research Reports}

\section{Determination of the Quantity of Viral RNAs by Northern Blotting}

CMV RNAs extracted from virions that had been tested previously by real-time RT-PCR were also tested by Northern hybridization according to standard procedures (16). RNAs 1, 2, and 3 were detected by a common conserved-40 probe, containing a sequence $\left(5^{\prime}\right.$-ACT GACCATTTTAGCCGTAAGCTGGA TTGGACAACCCGTTC-3') complementary to a conserved sequence present in the $3^{\prime}$ terminal of genomic RNAs in all Cucumoviruses. The probe was prepared by end-labeling of DNA oligonucleotide with $\gamma^{3}$-32P-ATP using $\mathrm{T}_{4}$ polynucleotide kinase (Takara). The amounts of CMV $1 a$, $2 a$, and $M P$ genes were represented by the hybridization intensity of RNAs 1, 2, and 3 bands, respectively.

\section{RESULTS}

\section{Quantification of CMV RNAs in Virions with Standard Curves}

Using standard samples, the standard curves of $1 a, 2 \mathrm{a}$, and $M P$ genes were constructed over a range of five orders of magnitude. As shown in Figure 1, these standard curves demonstrate a strong linear relation $\left(R^{2}>0.99\right)$ between the $C_{1}$ values and the log-copy numbers of CMV genes. The standard deviations in $\mathrm{C}_{\mathrm{T}}$ values are depicted as error bars in Figure 1, which were found to range from a minimum of 0.04 to a maximum of 0.79 cycles.

Based on the constructed standard curves, copy numbers of CMV RNAs 1,2 , and 3 in five types of virions were calculated. The lowest accumulation levels were found in FRad352b-CMV virions, and the highest levels in $\mathrm{FNa}^{2 \mathrm{~b}}-\mathrm{CMV}$ virions (Figure 2A). The accumulation ratios present in Fny-CMV, FCb72b_ CMV, FRad352b-CMV, FBX ${ }^{2 b}-C M V$, and $\mathrm{FNa}^{2 \mathrm{~b}} \mathrm{CMV}$ virions were calculated to be $1: 1.34: 2.36,1: 1.37: 2.17,1: 1.39: 1.47$, $1: 0.80: 2.20$, and $1: 1.38: 1.65$, respectively.

\section{Quantification of CMV RNAs in Virions by SCF}

As the starting procedure for quantitative assessment, the calculated $F_{0}$ values of a series of diluted standard samples were examined. As shown in Figure 3A, the relationship between $F_{0}$ and the initial concentration of $2 a$ gene is precisely linear with a correlation coefficient of 0.992 and $\mathrm{R}^{2}>0.999$. Similar results were obtained for $l a$ and $M P$ genes. Based on these $F_{0}$ values, the average $C F_{\mathrm{SCF}}$ were determined to be $61.2,65.8$, and $68.3 \mathrm{ng} / \mathrm{FU}$ for $1 a, 2 a$, and $M P$ genes, respectively (Table 1).

Using the determined $C F_{\text {SCF }}$ values, the copy numbers of CMV RNAs 1, 2 , and 3 in virions were calculated by Equation 4 (Figure 2B). As expected, the results were very similar to those obtained by the standard curve method, and their ratios were determined to be 1:1.22:2.40, 1:1.35:2.52, 1:1.49:1.66, 1:0.99:2.30, and 1:1.31:1.67.

\section{Quantification of CMV RNAs in Virions by LinRegPCR and DART}

When the experimentally derived fluorescence readings of the same realtime PCR procedure were treated by LinRegPCR and DART, a good linear relationship was also found between the $F_{0}$ values and concentration of standard

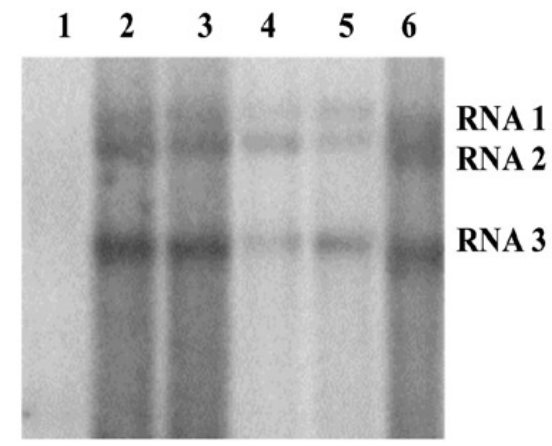

Figure 4. Quantification of the accumulation ratios of Cucumber mosaic virus (CMV) RNAs 1, 2, and 3 in five types of virions by Northern blotting. Lane 1, RNA extracted from mocked infection plants; lanes 2-6, RNA extracted from virions of Fny-CMV, FCb72b-CMV, FRad352b-CMV, FBX ${ }^{2} \mathrm{~b}_{-} \mathrm{CMV}$, and $\mathrm{FNa}^{2 \mathrm{~b}}-\mathrm{CMV}$ respectively.

samples of $2 a$ gene, as the results show in Figure 3B and Figure 3C.

Using the LinRegPCR program, the average $C F_{\text {LinReg }}$ values were determined to be $43.9,44.1$ and $47.9 \mathrm{ng} / \mathrm{FU}$ for $1 a$, $2 a$ and $M P$ genes (Table 1). These values were lower than those obtained by SCF. However, as shown in Figure 2C, such a decrease in $C F$ values has no significant effect on the final calculated results. The copy number ratios of RNAs 1,2 , and 3 in Fny-CMV, FCb72b-CMV, FRad352bCMV, $\mathrm{FBX}^{2 \mathrm{~b}}{ }_{-} \mathrm{CMV}$, and $\mathrm{FNa}^{2 \mathrm{~b}_{-} \mathrm{CMV}}$ virions were determined to be 1:1.35:2.39, 1:1.46:2.69, 1:1.60:1.51, 1:0.75:2.26, and 1:1.43:1.62, respectively.

With DART, $C F_{\text {DART }}$ values of $1 a, 2 a$, and $M P$ genes were determined to be 41.3 , 42.5 , and $49.1 \mathrm{ng} / \mathrm{FU}$, which are very close to the $C F_{\text {LinReg }}$ values (Table 1). Their variations $(15.93 \%, 11.79 \%$, and $12.28 \%$ for $1 a, 2 a$, and $M P$ genes, respectively), although significant, indicate that the DART approach has an acceptable level
A

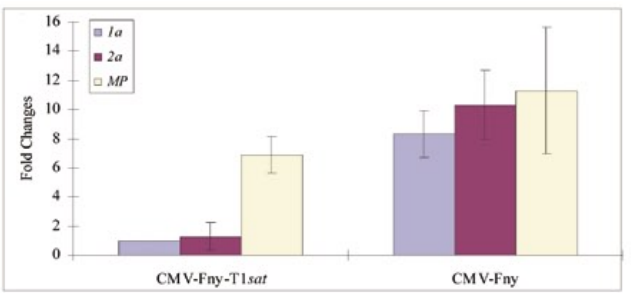

B

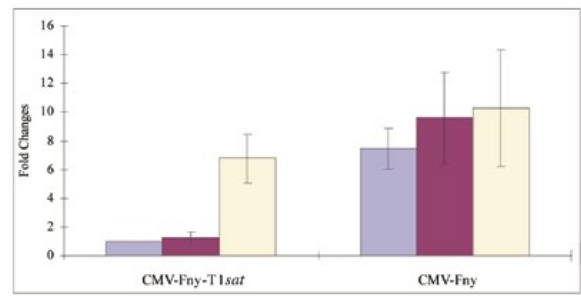

C

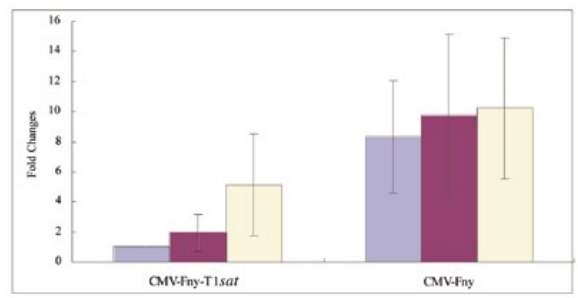

Figure 5. Relative comparison of the expression levels of Cucumber mosaic virus (CMV) RNAs in planta. The expression levels of CMV RNAs 1, 2, and 3 were determined by (A) sigmoidal curve-fitting, (B) LinRegPCR, and (C) DART methods. The amount of RNA 1 in CMV-Fny-T1sat infection was set at 1 and the other RNAs were quantified relative to it. As an endogenous control to normalize the data for differences in input total RNA between samples, 18S rRNA was used. The error bars indicate the standard deviations obtained from three independent experiments. 


\section{Research Reports}

of reproducibility over the five magnitudes of target concentration that we examined. Similarly, the copy numbers of CMV RNAs in five types of virions were calculated and their ratios were determined to be $1: 1.20: 2.43,1: 1.50: 2.26,1: 1.71: 1.53$, 1:0.86:2.34, and 1:1.57:1.40 (Figure 2D).

\section{Quantification of CMV RNAs in Virions by Northern Blotting and Comparison with Real-time PCRs}

To test the accuracy of the quantification results obtained from real-time PCRs, the accumulation ratios of CMV RNAs 1, 2, and 3 in virions were also determined by Northern blotting. Based on the hybridization intensity of each band (Figure 4), their ratios in Fny-CMV, FCb72b_CMV, FRad352b-CMV, FBX ${ }^{2 b}$ $\mathrm{CMV}$, and $\mathrm{FNa}^{2 \mathrm{~b}} \mathrm{CMV}$ virions were determined to be $1: 1.43: 2.23,1: 1.42: 2.13$,
1:1.69:1.66, 1:1.11:2.19, and 1:1.38:1.57, respectively.

When the results of all these quantification methods are compared, the SCF and the LinRegPCR program display a higher level of accuracy, as reflected by the low variance in determination of both $C F$ values and absolute copy numbers of viral genes (Table 1, Figures 2 and 3 ). The variance of results of Northern blotting cannot be calculated because no replicate measurements were performed. Nevertheless, the relative ratios of $1 a, 2 a$, and $M P$ genes in virions determined by all these methods are comparable (Table 2).

\section{Determination of the Suppression Effect of T1sat on CMV RNAs Accumulation in Plant Tissues Using $N_{0}$ Values}

Using Equations 9 and 10, the different accumulation levels of CMV RNAs 1, 2,
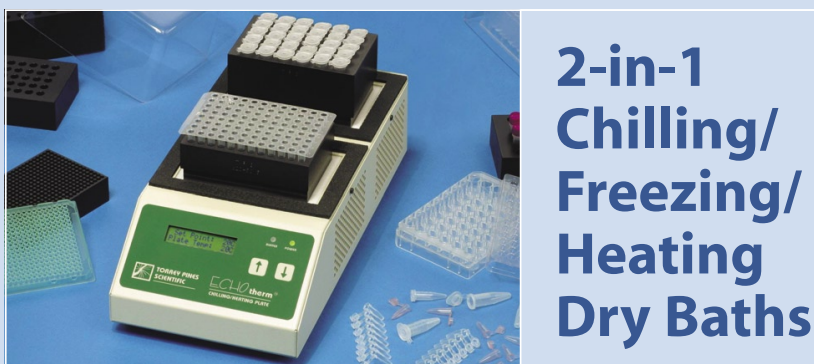

2 Dry Baths, 2 Sets of Sample Blocks, 2 Different Temperatures From $-20^{\circ} \mathrm{C}$ To $100^{\circ} \mathrm{C}$. The EchoTherm ${ }^{\mathrm{mm}}$ Model IC22

- $1^{\circ} \mathrm{C}$ resolution and control

- Two 30-day count down timers

- Two data loggers with RS232 I/O port

- Forty-plus standard sample blocks

- Custom blocks available to fit any sample holder

- Peltier based. Compact, reliable, and low energy

- 13 other models including shaking and programmable

- UL, CSA, and CE compliant

EchoTherm Chilling/Heating Dry Baths Are Ideal For:

- Enzyme reactions and deactivations - Hybridizations

- Ligations at $14^{\circ} \mathrm{C}$

PCR sample prep

- Storing Oocytes at $17^{\circ} \mathrm{C}$

- Incubations above, below or exactly at room temperature

- General molecular biology

TORREY PINES SCIENTIFIC, INC.

Tel: 760-471-9100 • Fax: 760-471-9310

email: info@torreypinesscientific.com

www.torreypinesscientific.com and 3 in tobacco tissues were detected at 4 dpi. As the results show in Figure 5, the average accumulation level ratios were calculated to be 1:0.98:1.16 in CMVFny infection. While in CMV-Fny-T1sat infection, the ratios changed to 1:1.49:6.27 and the accumulation levels of RNAs 1 , 2 , and 3 were $8.27,5.45$, and 1.53 folds lower than that of CMV-Fny infection, respectively. These ratios indicate that at this time point, the accumulation of all CMV genomic RNAs in tobacco were suppressed by T1sat, but the suppression effect on RNAs 1 and 2 was more apparent than on RNA 3.

The results demonstrate that using the $N_{0}$ values, a relative comparison between genes can be easily performed in the same sample or between different samples. When the relative ratios determined by these various methods are compared, the higher levels of accuracy of SCF and the LinRegPCR program are indicated by the smaller variations (Figure 5).

\section{DISCUSSION}

An accurate, precise, and facile calculation method is important for quantification in the application of real-time PCR (17). In the present study, we extend the work of Rutledge (3), by quantifying the expression levels of CMV RNAs with the conventional absolute approach but also with the more recent SCF, LinRegPCR, and DART methods. To our knowledge, this is the first report presenting such a comprehensive comparison between different real-time PCR mathematical models.

Although the standard curve method is broadly used for absolute quantification, the inherent complexity and imprecision associated with this method make interpretation difficult (18). In our study, the constructed standard curves of CMV genes showed high quality, for the linearity relationships between the log-concentration and $\mathrm{C}_{\mathrm{T}}$ values showed coefficient superior to 0.99 . However, according to previous studies $(7,19)$, it is not a good indicator of the precision or accuracy achieved, and should not be used to compare the quality of quantification results. Additionally, the overall accumulation levels of CMV RNAs 1, 2, and 3 determined by standard curve method were lower than those obtained by other 


\section{Research Reports}

methods. These are believed to be caused by overlooking the variations of PCR efficiency between standard and tested samples.

When the same data were used in SCF, LinRegPCR, and DART methods, we found that the last two are easier to use. With the DART program, the post-PCR data analysis process is remarkably facile, as all necessary calculations and statistical tests are performed automatically. However, the determined $C F_{\text {DARI }}$ values were found to be lower in our study when the concentrations of the standard samples were higher, which might be due to the presence of "inhibitor," therefore resulting in the lower $E$ values in these amplifications (14). In the linear regression analysis procedure, the log-linear part of the data points can be automatically determined, but a visual inspection is required to ensure that they are in the straight loglinear regime. Fitting the fluorescence data to a four-parametric sigmoidal function was proposed to represent the PCR reaction more effectively than using other functions (20). This method, however, requires extensive analyses, involving a curve-fitting process to select the cutoff cycle, beyond which any remaining cycles are excluded from the regression analysis. This delimitation procedure is required for every amplification and is therefore time consuming. Nevertheless, this enables the determination of results with small variability as is shown in this study. Therefore, considering both the accuracy of results and the procedure of data processing, the LinRegPCR method demonstrates a higher level of efficiency.

Theoretically, if it is assumed that length and base pair composition do not significantly influence DNA fluorescence, the relationship between $F_{0}$ and $N_{0}$ is solely dependent on product size, and the $C F$ value for each gene should accordingly be equal. However, recent publications have reported the preferential binding of SYBR Green I to specific sequences, but this process is complicated, and is significantly influenced by the ratio of base pair/dye molecule complexes and by the concentration of salts in reaction solution $(21,22)$. For the CMV $1 a, 2 a$, and $M P$ genes studied in this paper, the average ratio of their $C F$ values determined by the three different mathematical models was 1:1.04:1.13. However, not only are their $\mathrm{G}+\mathrm{C}$ contents $(49.3 \%, 47.35 \%, 47.85 \%)$ and product sizes (72 bp, $76 \mathrm{bp}, 154$ bp) different, but also the concentration of SYBR Green in commercial kits is unknown, thus the variations in $C F$ values are difficult to explain and require further detailed systematic studies.

The $C F$ values determined in this study have important practical implications. First, they could be used to calculate the definitive copy numbers of viral genes without the construction of standard curves for each target sequence, thus allowing large numbers of different targets to be simultaneously quantified. Secondly, the variations of RT and PCR efficiency between target genes would be circumvented, thus either the normalization or relative comparison between genes can be derived without assumptions.

Northern blotting is a conventional approach for quantifying CMV genes due to the simple linear relationship between the intensity of each band and its quantity. However, Northern blotting often fails to provide reliable results because the hybridization rates are different for each probe. In this study, this problem was overcome by the use of a common probe in the detection of CMV genes. Although the definitive copy numbers of each gene in the virions could not be calculated, the ratios of their amounts determined by hybridization intensity were very similar to those obtained from real-time PCR data.

CMV-encoded $2 \mathrm{~b}$ proteins have been shown to play an important role in viral long-distance movement, hypervirulence, and suppression of post-transcriptional gene silencing. Our previous work showed that a change of CMV virulence resulted from different accumulation of viral RNAs, which is induced by either replacement of $2 b$ ORFs or by addition of satRNAs $(10,11)$. In this study, we propose equations to quantify the accumulation ratios of CMV RNAs 1, 2, and 3 in the same sample or between different samples, by analyzing the results of real-time PCR using SCF, LinRegPCR, and DART methods. The high-throughput quantification ability of real-time PCR would be enhanced by the use of these equations, as the need for standard curves is eliminated, enabling large numbers of targets to be analyzed simultaneously. Among them, the LinRegPCR method is recommended by us for the reliable results it provides and for the simplified calculation it involves.
In conclusion, the equations we proposed, with the $F_{0}$ and $C F$ values determined by LinRegPCR, can be used as a more convenient approach for quantifying and comparing the relative accumulation levels of CMV RNAs. They should be especially useful for investigating the mechanism of $\mathrm{CMV}$ replication in host plants and for determining CMV resistance levels in novel genetically engineered plants.

\section{ACKNOWLEDGMENTS}

We would like to thank Greg Duns who carefully read and corrected the manuscript. This work was supported by the Chinese National Key Project for International Cooperation (no. 2004DFA05000) and Chinese National Foundation of Science (no. 30671361).

\section{COMPETING INTERESTS STATEMENT}

The authors declare no competing interests.

\section{REFERENCES}

1. Valasek, M.A. and J.J. Repa. 2005. The power of real-time PCR. Adv. Physiol. Educ. 29:151-159.

2. Mackay, I.M., K.E. Arden, and A. Nitsche. 2002. Real-time PCR in virology. Nucleic Acids Res. 30:1292-1305.

3. Rutledge, R.G. 2004. Sigmodial curve-fitting redefines quantitative real-time PCR with the prospective of developing automated high-throughput applications. Nucleic Acids Res. 32:e178.

4. Wilhelm, J., A. Pingoud, and M. Hahn. 2003. SoFAR: software for fully automatic evaluation of real-time PCR data. BioTechniques 34:324-332.

5. Dheda, K., J.F. Huggett, J.S. Chang, L.U. Kim, S.A. Bustin, M.A. Johnson, and A. Zumla. 2005. The implications of using an inappropriate reference gene for real-time reverse transcription PCR data normalization. Anal. Biochem. 344:141-143.

6. Pfaffl, M.W. 2001. A new mathematical model for relative quantification in real-time RT-PCR. Nucleic Acids Res. 29:e45.

7. Rutledge, R.G. and C. Côté. 2003 Mathematics of quantitative kinetics PCR and the application of standard curves. Nucleic Acids Res. 31:e93.

8. Palukaitis, P. and F. Garíca-Arenal. 2003 Cucumoviruses. Adv. Virus Res. 62:241-323.

9. Feng, J.L., S.N. Chen, X.S. Tang, X.F. Ding, Z.Y. Du, and J.S. Chen. 2006. Quantitative 


\section{Research Reports}

determination of Cucumber mosaic virus genomic RNAs in virions by real-time reverse transcription-polymerase chain reaction. Acta Bioch Bioph Sin. 38:669-676.

10. Liao, Q., L. Zhu, Z. Du, R. Zeng, and J. Chen. 2007. Satellite RNA-mediated reduction of Cucumber mosaic virus genomic RNAs accumulation in Nicotiana tabacum. Acta Bioch. Bioph. Sin. 39:217-223.

11. Du, Z.Y., F.F. Chen, Q.S. Liao, H.R. Zhang, Y.F. Chen, and J.S. Chen. 2007. 2b ORFs encoded by subgroup IB strains of Cucumber mosaic virus induce differential virulence on Nicotiana species. J. Gen. Virol. 88:25962604

12. Liu, W. and D.A. Saint. 2002. Validation of a quantitative method for real time PCR kinetics. Biochem. Biophys. Res. Commun. 294:347-353.

13. Ramakers, C., J.M. Ruijter, R.H. Deprez, and A.F. Moorman. 2003. Assumption-free analysis of quantitative real-time polymerase chain reaction (PCR) data. Neurosci. Lett. 339:62-66.

14. Peirson, S.N., J.N. Butler, and R.G. Foster. 2003. Experimental validation of novel and conventional approaches to quantitative realtime PCR data analysis. Nucleic Acids Res. 31:e73.
15. Livak, K.J. 1997. ABI Prism 7700 Sequence Detection System, User Bulletin 2, PE Applied Biosystems.

16. Sambrook, J. and D.W. Russell. 2001. Molecular Cloning: A Laboratory Manual, p. 517-589. 3rd ed. Science Publishing House, Beijing, China.

17. Bar, T., A. Stahlberg, A. Muszta, and M. Kubista. 2003. Kinetic Outlier Detection (KOD) in real-time PCR. Nucleic Acids Res. 31:e105.

18. Qiu, H., K. Durand, H.R. Chable, M. Rigaud, V. Gazaille, P. Clavere, and F.G. Sturtz. 2007. Gene expression of HIF-1alpha and XRCC4 measured in human samples by real-time RT-PCR using the sigmoidal curvefitting method. BioTechniques 42:355-362.

19. Wilhelm, J., A. Pingoud, and M. Hahn. 2003. Validation of an algorithm for automatic quantification of nucleic acid copy numbers by real-time polymerase chain reaction. Anal. Biochem. 317:218-225.

20. Liu, W. and D.A. Saint. 2002. A new quantitative method of real time reverse transcription polymerase chain reaction assay based on simulation of polymerase chain reaction kinetics. Anal. Biochem. 302:52-59.

21. Giglio, S., P.T. Monis, and C.P. Saint. 2003. Demonstration of preferential binding of SYBR Green I to specific DNA fragments in real-time multiplex PCR. Nucleic Acids Res 31:e136.

22. Zipper, H., H. Brunner, J. Bernhagen, and F. Vitzthum. 2004. Investigations on DNA intercalation and surface binding by SYBR Green I, its structure determination and methodological implications. Nucleic Acids Res. 32:e103.

Received 2 November 2007; accepted 18 December 2007.

Address correspondence to Jishuang Chen, Institute of Bioengineering, Zhejiang Sci-Tech University, Xiasha Road, \#2, Hangzhou, 310018, P.R. China. e-mail: chenjs@zist.edu.cn

To purchase reprints of this article, contact: Reprints@BioTechniques.com

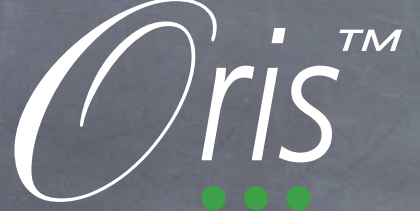

Kinetic Analysis

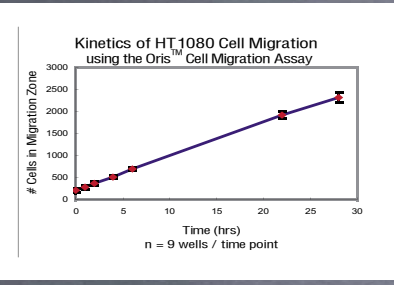

\section{BENEFITS of ORIS TM CELL MIGRATION ASSAY}

- Membrane-FrEE Migration: no transwell inserts

- Seed Cells and Read Results All in One well

- No Need to Separate, Lyse, and Transfer Cells

- Highly Reproducible Results

- Analysis Via Microscope or Fluorescence Plate Reader

Endpoint Analysis
Cell Migration

1. Seed and Adhere Cells onto OrisTM Plate

.

\section{Remove Stopper to Create Migration Zone}

3. Incubate and Allow Cells to Migrate

\section{Analyze Migration Zone}
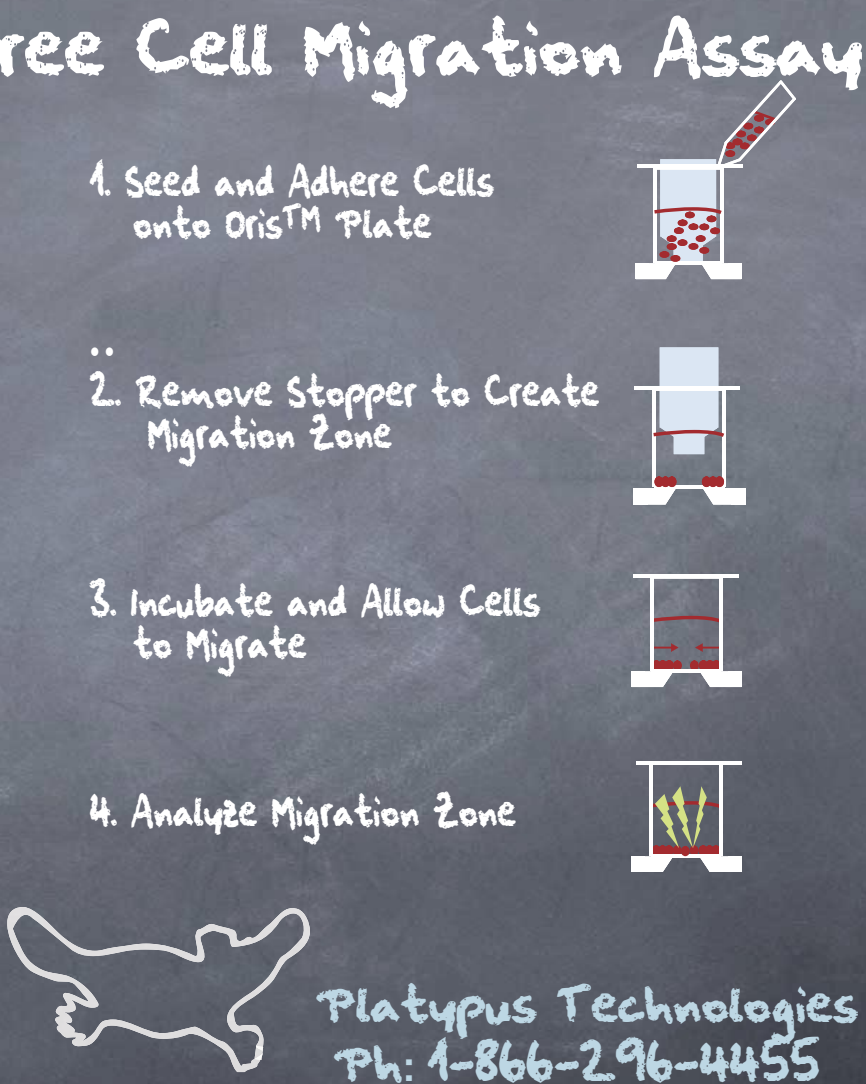

Platypus Technologies Ph: $1-366-296-4455$ 